\title{
CME Cardiovascular medicine (129460): self-assessment
} questionnaire

\author{
Authors: Edited by Tevfik F Ismail and Tahseen A Chowdhury
}

SAQs and answers are ONLINE for RCP fellows and collegiate members

\section{Format}

Candidates are asked to choose the best answer from the five possible answers. This best of five format is used in many medical examinations; however, the questions are not intended to be representative of those used in the MRCP(UK) Part 1 or Part 2 Written Examinations.

\section{The answering process}

1 Go to www.rcplondon.ac.uk/SAQ

2 Log on using your usual RCP username and password

3 Select the relevant CME question paper

4 Answer all 10 questions by selecting the best answer from the options provided

5 Once you have answered all the questions, click on Submit

\section{Registering your external CPD credits}

Carrying out this activity allows you to claim two external CPD credits. These will be automatically transferred to your CPD diary, where you can review the activity and claim your points.

1. A 26-year-old man was admitted with a 2-day history of pleuritic left-sided chest pain. Acute pericarditis was considered.

\section{Which of the following clinical features is not a diagnostic} criterion for acute pericarditis?
(a) chest pain
(b) fever
(c) pericardial effusion
(d) pericardial rub
(e) widespread ST-elevation and/or PR-depression.

2. A 53-year-old woman was seen in the emergency department with a 2-week history of increasing breathlessness, and left-sided pleuritic chest pain. Electrocardiography showed widespread ST-elevation, and acute pericarditis was considered.

\section{Which of the following clinical features should not necessarily prompt consideration of hospital admission?}

(a) a $22 \mathrm{~mm}$ pericardial effusion on echo

(b) a temperature of $38.1^{\circ} \mathrm{C}$

(c) abrupt/rapid onset of symptoms

(d) concomitant treatment with warfarin

(e) raised jugular venous pressure with quiet heart sounds on auscultation.

3. A 32-year-old woman was found to have a low-density lipoprotein cholesterol of $5.8 \mathrm{mmol} / \mathrm{L}$. She had a strong family history of elevated cholesterol and her father suffered a myocardial infarction at the age of 42 years.

Which of the following statements concerning familial hypercholesterolaemia is correct?

(a) diagnosis should be confirmed by genetic testing

(b) ezetimibe has no role in therapy

(c) inheritance is autosomal recessive

(d) it affects 1 in 600 of the population

(e) it should be treated with a proprotein convertase subtilisin kexin 9 inhibitor.

4. A 51-year-old man was admitted with an acute ST elevation myocardial infarction and was due to be commenced on statin therapy.

\section{Which of the following statements concerning statin} therapy is correct?
(a) atorvastatin $20 \mathrm{mg}$ nocte is a reasonable choice for secondary prevention patients
(b) creatine kinase should be measured pre-and post initiation
(c) intolerance is defined as the inability to take five different statins
(d) should be replaced by ezetimibe in patients who are genuinely statin intolerant
(e) there is no need to check liver function tests prior to initiation. 
5. A 26-year-old woman was seen in the emergency department with acute palpitations. Electrocardiography showed a narrow complex tachycardia.

In the acute management of a narrow complex tachycardia, which of the following statements is correct?

(a) adenosine is absolutely contraindicated in patients with a history of asthma

(b) atrial tachycardia is the likely diagnosis if successfully terminated with adenosine

(c) beta-blockers are highly efficacious at terminating supraventricular tachycardias

(d) calcium channel blockers should be avoided in patients with a history of left ventricle dysfunction

(e) the mean dose of adenosine required for termination is $12 \mathrm{mg}$.

6. A 30-year-old man presented with palpitations to the emergency department. His rhythm strip is shown in the figure below.

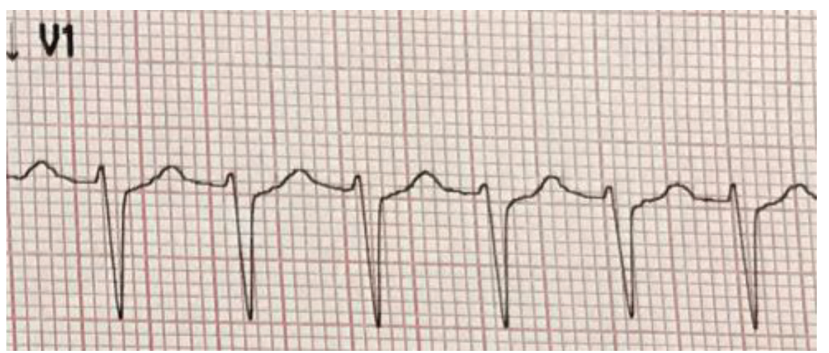

What is the likely arrhythmia?
(a) atrial fibrillation
(b) atrial tachycardia
(c) atrioventricular nodal re-entrant tachycardia
(d) atrioventricular re-entrant tachycardia
(e) sinus tachycardia.

7. Concerning pre-excited atrial fibrillation, which of the following is true?

(a) it is a medical emergency and adenosine should be used as first-line therapy

(b) it is characterised by an irregular rhythm with varying QRS duration

(c) it is often difficult to manage, and beta-blocker therapy should be used as first-line therapy

(d) it its occasionally seen in patients with atrioventricular nodal re-entrant tachycardia

(e) it should have routine specialist outpatient follow-up.
8. A 45-year-old woman presented with fevers and breathlessness. She was noted to have an aortic murmur and some stigmata of endocarditis.

Concerning endocarditis, which of the following statements is correct?

(a) acute endocarditis is the commonest presentation of the condition

(b) endocarditis usually affects individuals $<50$ years of age

(c) enterococci are the commonest cause of endocarditis in industrialised nations

(d) most cases of endocarditis are community acquired

(e) with improved diagnostic imaging and microbiological techniques, the rates of endocarditis are decreasing globally.

9. Regarding the use of echocardiography in establishing a diagnosis of endocarditis, which of the following statements is correct?

(a) a mobile mass found on echocardiography is diagnostic for endocarditis

(b) it is reasonable to repeat transthoracic echocardiography in a patient with suspected infective endocarditis within 1 week if the first scan was normal

(c) normal transthoracic echocardiography excludes a diagnosis of endocarditis

(d) transoesophageal echocardiography should be performed in all patients with suspected endocarditis

(e) transthoracic echocardiography should be immediately performed in all patients with a pyrexia and heart murmur.

10. Which of the following is an established indication for emergency cardiac surgery in infective endocarditis?
(a) aortic pseudoaneurysm
(b) aortic root abscess
(c) cardiogenic shock secondary to valve disease/sepsis
(d) enlarging vegetation
(e) uncontrollable infection.

\section{CME Diabetes Medicine SAQ Answers to the CME SAQ published in Clinical Medicine in September 2019
Q1 Q2
Q3
Q4 Q5
Q6 Q7
Q8 Q9
(e) (e) (c)
(d) (b)
(b) (a)
(c) (d)
(b) (b) \\ Q10}


Royal College

of Physicians

\title{
Complex regional pain syndrome in adults
}

\author{
UK guidelines for diagnosis, \\ referral and management in \\ primary and secondary care
}

Published by the Royal College of Physicians, these guidelines concern the diagnosis and management of patients with complex regional pain syndrome (CRPS). They are designed for professionals working in the different health specialties who care for these patients.

Updated in 2018, and published in partnership with over 20 other medical organisations, the guidelines provide information on diagnosis, treatment, management and support for patients in a wide variety of clinical settings.

The report underlines the need for multidisciplinary support to manage CRPS and outlines the four pillars of care that underpin management:

$>$ education

$>$ pain relief

$>$ physical rehabilitation

$>$ psychological intervention.

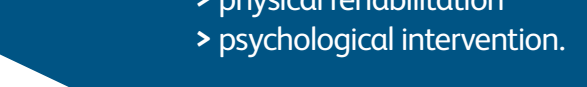

